\title{
SOME RESTRICTED PARTITION FUNCTIONS: CONGRUENCES MODULO 2
}

\author{
BY \\ D. B. LAHIRI
}

1. Introduction. In an earlier communication [7] the author has established some congruence relations with respect to the modulus 7 for some restricted partition functions. In the present paper we shall prove congruences for the same type of functions but with 2 as the modulus. In the derivation of the results for these two moduli there is some parallelism in approach but there are important points of difference also.

As explained in the previous paper, the difference between the unrestricted partition function (cf. Gordon [2])

$$
{ }_{r}^{\alpha} p(n),
$$

merely lies in the restriction that no number of the forms $\alpha n$ or $\alpha n \pm r$ shall be a part of the partitions which are of relevance in the restricted case. Thus to determine the value of ${ }_{r}^{\alpha} p(n)$ one shall count all the unrestricted partitions of $n$ excepting those which contain a number of any of the above forms as a part.

We now state our main results. The phrase "for almost all values of $n$ " appearing in connection with certain congruences in this paper means that the number of integers $n \leqq N$ for which the specified congruence does not hold is $o(N)$.

THEOREM 1. For almost all values of $n$

$$
\begin{array}{ll}
{ }_{14}^{48} p(n) \equiv{ }_{2}^{48} p(n-4) & (\bmod 2), \\
{ }_{22}^{48} p(n) \equiv{ }_{10}^{48} p(n-2) & (\bmod 2) .
\end{array}
$$

THEOREM 2. For almost all values of $n$

$$
\begin{aligned}
& { }_{44}^{192} p(n) \equiv{ }_{20}^{192} p(n-8) \quad(\bmod 2), \\
& { }_{68}^{192} p(n) \equiv{ }_{4}^{192} p(n-20) \quad(\bmod 2), \\
& { }_{76}^{192} p(n) \equiv{ }_{52}^{192} p(n-4) \quad(\bmod 2), \\
& { }_{92}^{192} p(n) \equiv{ }_{28}^{192} p(n-12) \quad(\bmod 2) .
\end{aligned}
$$

2. Definitions and notations. We shall use $m$ to denote an integer, positive, zero or negative; but $n$ is reserved for a nonnegative integer only.

Received by the editors August 12, 1968 and, in revised form, July 14, 1969. 
Ramanujan [8] defined

$$
\Phi_{r, s}(x)=\sum_{\alpha=1}^{\infty} \sum_{\beta=1}^{\infty} \alpha^{r} \beta^{s} x^{\alpha \beta}=\sum_{n=1}^{\infty} n^{r} \sigma_{s-r}(n) x^{n},
$$

where $\sigma_{k}(n)$ is the sum of the $k$ th powers of the divisors of $n$. The author has found it convenient to simplify the notation to $\Phi_{r, s}$ in [5].

The function $u_{r, 0}(x)$, or simply $u_{r}$, used earlier by the author [5] is defined as follows.

$$
u_{r}=\sum_{n=0}^{\infty} n^{r} a_{n} x^{n} \cdot \sum_{n=0}^{\infty} p(n) x^{n}
$$

where $a_{n}$ is defined by the well-known "pentagonal number" theorem of Euler,

$$
f(x)=\prod_{n=1}^{\infty}\left(1-x^{n}\right)=\sum_{-\infty}^{+\infty}(-1)^{m} x^{m(3 m+1) / 2}=\sum_{n=0}^{\infty} a_{n} x^{n}
$$

and $p(n)$ is the number of unrestricted partitions of $n$ given by the expansion,

$$
[f(x)]^{-1}=\left[\prod_{n-1}^{\infty}\left(1-x^{n}\right)\right]^{-1}=\sum_{n=0}^{\infty} p(n) x^{n} .
$$

We shall use $v$ to denote the pentagonal numbers,

$$
v=m(3 m+1) / 2, \quad m=0, \pm 1, \pm 2, \ldots ;
$$

and with each $v$ there corresponds an "associated sign", viz., $(-1)^{m}$. We shall come across sums of the type $\Sigma_{v}[\mp V(v)]$ where it is understood that the sign to be prefixed is the associated one. With this summation notation we can write

$$
u_{r}=\sum_{v}\left(\mp v^{r} x^{v}\right) / f(x),
$$

and Euler's theorem (3) as

$$
\sum_{v}\left(\mp x^{v}\right) / f(x)=1
$$

3. Some lemmas. We shall have to make use of the identities given in the following lemma. These identities have been established earlier, Lahiri [5, p. 128]. The first two identities are essentially due to Catalan [1, p. 290] and Glaisher [1, p. 312] respectively.

\section{LEMMA 1.}

$$
\begin{aligned}
u_{1}= & -\Phi_{0,1} \\
u_{2}= & (1 / 12) \Phi_{0,1}-(3 / 2) \Phi_{1,2}+(5 / 12) \Phi_{0,3} \\
u_{3}= & -(1 / 192) \Phi_{0,1}+(3 / 16) \Phi_{1,2}-(15 / 8) \Phi_{2,3} \\
& -(5 / 96) \Phi_{0,3}+(25 / 32) \Phi_{1,4}-(7 / 192) \Phi_{0,5}
\end{aligned}
$$


The truth of the following lemma can be easily verified by writing $4 m+j$ with $j=0,1$, and $-2,-1$ respectively in place of $m$ in the expression $m(3 m+1) / 2$ for the pentagonal number and in $(-1)^{m}$ its associated sign. It is to be remembered that $(1 / 2)\left(4 m-j^{\prime}\right)\left(12 m-3 j^{\prime}+1\right)$ and $(1 / 2)\left(4 m+j^{\prime}\right)\left(12 m+3 j^{\prime}-1\right)$ represent the same set of numbers.

LEMMA 2. The solutions of the following congruence in $v, v \equiv i(\bmod 2)$ and the corresponding associated signs are as follows.

\begin{tabular}{llclc}
$i$ & solutions $(1$ st set $):$ & sign & solutions $(2 n d$ set $):$ & sign \\
\hline 0 & $24 m^{2}+2 m$ & + & $24 m^{2}+14 m+2$ & - \\
1 & $24 m^{2}+22 m+5$ & + & $24 m^{2}+10 m+1$ & -
\end{tabular}

The truth of the next lemma can be similarly established by writing $8 m+j$ with $j=0, \pm 1, \pm 2, \pm 3,-4$ in place of $m$ in the expression for $v$ in terms of $m$.

LeMma 3. $v^{3}+v^{2}, v^{3}-v$ and $v^{3}-v^{2}$ are divisible by 4 excepting in the following cases,

$$
v^{3}+v^{2} \equiv 2(\bmod 4) \text { if } v \equiv 1(\bmod 4),
$$

i.e. when $v=96 m^{2}+20 m+1$, or $96 m^{2}+44 m+5$;

$$
v^{3}-v \equiv 2(\bmod 4) \quad \text { if } v \equiv 2(\bmod 4),
$$

i.e. when $v=96 m^{2}+28 m+2$, or, $96 m^{2}+92 m+22$;

$$
v^{3}-v^{2} \equiv 2(\bmod 4) \text { if } v \equiv 3(\bmod 4),
$$

i.e. when $v=96 m^{2}+52 m+7$, or $96 m^{2}+76 m+15$.

Taking into account the well-known case of a famous identity of Jacobi [4, p. 283] viz.

$$
\sum_{-\infty}^{+\infty}(-1)^{m} x^{k m^{2}+l m}=\prod_{n=0}^{\infty}\left[\left(1-x^{2 k n+k-l}\right)\left(1-x^{2 k n+k+l}\right)\left(1-x^{2 k n+2 k}\right)\right],
$$

and the easily established fact that

$$
\prod_{n=0}^{\infty}\left[\left(1-x^{\alpha n+r}\right)\left(1-x^{\alpha n+\alpha-r}\right)\left(1-x^{\alpha n+\alpha}\right)\right] / f(x)=\sum_{n=0}^{\infty}{ }_{r}^{\alpha} p(n) x^{n},
$$

and noting that

$$
\sum_{-\infty}^{+\infty} x^{k m^{2}+l m} \equiv \sum_{-\infty}^{+\infty}(-1)^{m} x^{k m^{2}+l m} \quad(\bmod 2),
$$

one easily obtains the next lemma.

LEMMA 4.

$$
\sum_{-\infty}^{+\infty} x^{k m^{2}+l m} \mid f(x) \equiv \sum_{n=0}^{\infty}{ }_{k-l}^{2 k} p(n) x^{n} \quad(\bmod 2)
$$


To derive the next lemma we start from the obvious relation

$$
\sum_{v}\left(\mp x^{v}\right)=\sum_{v \equiv 0}\left(\mp x^{v}\right)+\sum_{v \equiv 1}\left(\mp x^{v}\right)
$$

where we write $v \equiv i$ simply for $v \equiv i(\bmod 2)$. Then making use of Lemma 2 we obtain

$$
\begin{aligned}
\sum_{v}\left(\mp x^{v}\right)= & \sum_{-\infty}^{+\infty} x^{24 m^{2}+2 m}-x^{2} \sum_{-\infty}^{+\infty} x^{24 m^{2}+14 m} \\
& +x^{5} \sum_{-\infty}^{+\infty} x^{24 m^{2}+22 m}-x \sum_{-\infty}^{+\infty} x^{24 m^{2}+10 m}
\end{aligned}
$$

Now dividing both sides of (12) by $f(x)$ and remembering the relation (7) and Lemma 4 we obtain,

$$
1=\sum_{n=0}^{\infty}{ }_{22}^{48} p(n) x^{n}-x^{2} \sum_{n=0}^{\infty}{ }_{10}^{48} p(n) x^{n}+x^{5} \sum_{n=0}^{\infty}{ }_{2}^{48} p(n) x^{n}-x \sum_{n=0}^{\infty}{ }_{14}^{48} p(n) x^{n} .
$$

Hence equating to zero the coefficient of $x^{n}, n>0$, on the right-hand side we get Lemma 5.

LEMMA 5. $\quad{ }_{22}^{48} p(n)+{ }_{2}^{48} p(n-5)={ }_{14}^{48} p(n-1)+{ }_{10}^{48} p(n-2), \quad n>0$.

In precisely the same manner as above we can make use of the identity,

$$
\sum_{v}\left(\mp x^{v}\right)=\sum_{i=0}^{3} \sum_{v \equiv i}\left(\mp x^{v}\right)
$$

where $v \equiv i$ stands for $v \equiv i(\bmod 4)$, to obtain the next lemma.

LEMMA 6. If $n$ is positive then

$$
\begin{aligned}
{ }_{92}^{192} p(n)+{ }_{52}^{192} p(n-5) & +{ }_{44}^{192} p(n-7)+{ }_{4}^{192} p(n-22) \\
& ={ }_{76}^{192} p(n-1)+{ }_{68}^{192} p(n-2)+{ }_{28}^{192} p(n-12)+{ }_{20}^{192} p(n-15) .
\end{aligned}
$$

4. Proofs of Theorems 1 and 2. The proofs rest upon the following two basic theorems which we shall immediately establish.

THEOREM 0.1. The following congruences modulo 2 are true for $n>0$.

(i) ${ }_{14}^{48} p(n-1)-{ }_{2}^{48} p(n-5) \equiv \sigma(n)$;

(ii) ${ }_{22}^{48} p(n)-{ }_{10}^{48} p(n-2) \equiv \sigma(n)$.

THEOREM 0.2. The following congruences modulo 2 are true for $n>0$.

$$
\begin{aligned}
&{ }_{76}^{192} p(n-1)-{ }_{52}^{192} p(n-5) \\
& \equiv 2^{-7}\left[7 \sigma_{5}(n)+2(53 n-35) \sigma_{3}(n)+\left(104 n^{2}-4 n-15\right) \sigma(n)\right] \\
&{ }_{68}^{192} p(n-2)-{ }_{4}^{192} p(n-22) \\
& \equiv 2^{-7}\left[7 \sigma_{5}(n)+2(53 n+5) \sigma_{3}(n)+\left(104 n^{2}-36 n+65\right) \sigma(n)\right]
\end{aligned}
$$


(iii)

$$
\begin{aligned}
& { }_{44}^{192} p(n-7)-{ }_{20}^{192} p(n-15) \\
& \equiv 2^{-7}\left[7 \sigma_{5}(n)+2(53 n+45) \sigma_{3}(n)+\left(104 n^{2}-68 n+17\right) \sigma(n)\right] ; \\
& { }_{92}^{192} p(n)-{ }_{28}^{192} p(n-12) \\
& \equiv 2^{-7}\left[7 \sigma_{5}(n)+2(53 n+53) \sigma_{3}(n)+\left(104 n^{2}+28 n+33\right) \sigma(n)\right] .
\end{aligned}
$$

The first identity of Lemma 1 coupled with the relation (6) for $r=1$ shows that

$$
\sum_{v}\left(\mp v x^{v}\right) / f(x)=-\Phi_{0,1}
$$

An immediate consequence is

$$
\sum_{v \equiv 1(\bmod 2)}\left(\mp x^{v}\right) / f(x) \equiv-\Phi_{0,1} \quad(\bmod 2) .
$$

Now, remembering Lemma 2 we get

$$
\left[x^{5} \sum_{-\infty}^{+\infty} x^{24 m^{2}+22 m}-x \sum_{-\infty}^{+\infty} x^{24 m^{2}+10 m}\right] / f(x) \equiv-\Phi_{0,1} \quad(\bmod 2) .
$$

Applying Lemma 4 to the above relation and comparing the coefficients of $x^{n}$, $n>0$, on both sides we obtain the first congruence of Theorem 0.1 . This congruence considered along with Lemma 5 leads to other congruence of this theorem. Thus Theorem 0.1 is fully established.

We now turn to Theorem 0.2. Eliminating $u_{r}$ 's between (6) and Lemma 1 we obtain three identities, and the following congruences are very trivial deductions therefrom.

$$
\begin{array}{rlr}
\sum_{v}\left[\mp\left(v^{3}+v^{2}\right)\right] / f(x) \equiv & (5 / 64) \Phi_{0,1}-(21 / 16) \Phi_{1,2}-(15 / 8) \Phi_{2,3} & \\
& +(35 / 96) \Phi_{0,3}+(25 / 32) \Phi_{1,4}-(7 / 192) \Phi_{0,5} \quad(\bmod 4) ; \\
\sum_{v}\left[\mp\left(v^{3}-v\right)\right] / f(x) \equiv & (191 / 192) \Phi_{0,1}+(3 / 16) \Phi_{1,2}-(15 / 8) \Phi_{2,3} & \\
& -(5 / 96) \Phi_{0,3}+(25 / 32) \Phi_{1,4}-(7 / 192) \Phi_{0,5} & (\bmod 4) ; \\
\sum_{v}\left[\mp\left(v^{3}-v^{2}\right)\right] / f(x) \equiv & -(17 / 192) \Phi_{0,1}+(27 / 16) \Phi_{1,2}-(15 / 8) \Phi_{2,3} & \\
& -(15 / 32) \Phi_{0,3}+(25 / 32) \Phi_{1,4}-(7 / 192) \Phi_{0,5} \quad(\bmod 4) .
\end{array}
$$

With the help of Lemmas 3 and 4 the left-hand sides of (18), (19) and (20) may be replaced respectively by

$$
\begin{gathered}
-2 x \sum_{n=0}^{\infty}{ }_{{ }_{76}}^{192} p(n) x^{n}+2 x^{5} \sum_{n=0}^{\infty}{ }_{52}^{192} p(n) x^{n} \\
-2 x^{2} \sum_{n=0}^{\infty}{ }_{68}^{192} p(n) x^{n}+2 x^{22} \sum_{n=0}^{\infty}{ }_{4}^{192} p(n) x^{n} \\
2 x^{7} \sum_{n=0}^{\infty}{ }_{44}^{192} p(n) x^{n}-2 x^{15} \sum_{n=0}^{\infty}{ }_{20}^{192} p(n) x^{n}
\end{gathered}
$$


Now comparing the coefficients of $x^{n}, n>0$, on both sides of (18), (19) and (20) we obtain the first three congruences of Theorem 0.2. To establish the last congruence (iv) of this theorem we make use of Lemma 6 and these three congruences (i)-(iii) in the obvious manner.

Now Theorems 1 and 2 are almost immediate consequences of Theorems 0.1 and 0.2 when one remembers the well-known congruence, $[9,3, \mathrm{p} .167]$,

$$
\sigma_{k}(n) \equiv 0 \quad(\bmod M) \text { for almost all values of } n,
$$

for arbitrarily fixed $M$ and odd $k$; the phrase "almost all values of $n$ " is in the sense explained in the introductory section. The passage from Theorem 0.1 to Theorem 1 needs the special case $k=1, M=2$ of the relation (21). But for the other theorem we make use of the cases $k=1,3,5$ with $M=2^{8}$, in Theorem 0.2.

5. Further results. The results given in the last section lead to certain interesting corollaries.

COROLLARY 1. The members of each of the following pairs have the same parity unless $n$ is a square or twice a square.

$$
\begin{array}{r}
{ }_{14}^{48} p(n-1) \text { and } \quad{ }_{2}^{48} p(n-5) ; \\
{ }_{22}^{48} p(n) \text { and } \quad{ }_{10}^{48} p(n-2)
\end{array}
$$

COROLlaRY 2. For given $n$, the members of each of the following pairs are either always of the same parity or always of opposite parity within the entire range $\lambda \geqq 0$.

$$
\begin{array}{r}
{ }_{76}^{192} p\left(2^{\lambda+7} n-1\right) \quad \text { and } \quad{ }_{52}^{192} p\left(2^{\lambda+7} n-5\right) \\
{ }_{68}^{192} p\left(2^{\lambda+7} n-2\right) \quad \text { and } \quad{ }_{4}^{192} p\left(2^{\lambda+7} n-22\right) ; \\
{ }_{44}^{192} p\left(2^{\lambda+7} n-7\right) \quad \text { and } \quad{ }_{20}^{192} p\left(2^{\lambda+7} n-15\right) ; \\
{ }_{92}^{192} p\left(2^{\lambda+7} n\right) \text { and } \quad{ }_{28}^{192} p\left(2^{\lambda+7} n-12\right)
\end{array}
$$

In connection with the above corollaries it may be explained that two numbers $\alpha$ and $\beta$ are said to be of the same parity if both are even or both are odd; and they are said to be of opposite parity when one is even and the other is odd.

Theorem 0.1 gives rise easily to the first corollary when considerations are given to the question of divisibility of $\sigma(n)$ by 2 . Derivation of Corollary 2 from Theorem 0.2 , however, is not quite so straightforward. We shall have to make use of the following easily proved congruence.

$$
\sigma_{k}\left(2^{\lambda+7} n\right) \equiv \sigma_{k}\left(2^{7} n\right) \quad\left(\bmod 2^{8 k}\right), \quad k \geqq 1 .
$$

As an illustration we shall show how (22) can be used to establish (iv) of Corollary 2 
from (iv) of Theorem 0.2. In this last congruence we write $2^{\lambda+7} n$ in place of $n$, and then make use of (22) to establish the following.

$$
\begin{aligned}
2^{7}\left[{ }_{92}^{192} p\left(2^{\lambda+7} n\right)-{ }_{28}^{192} p\left(2^{\lambda+7} n-12\right)\right] & \\
\equiv & 7 \sigma_{5}\left(2^{\lambda+7} n\right)+2\left(53.2^{\lambda+7} n+53\right) \sigma_{3}\left(2^{\lambda+7} n\right) \\
& \quad+\left(104.2^{2 \lambda+14} n^{2}+28.2^{\lambda+7} n+33\right) \sigma\left(2^{\lambda+7} n\right) \\
\equiv & 7 \sigma_{5}\left(2^{7} n\right)+106 \sigma_{3}\left(2^{7} n\right)+33 \sigma\left(2^{7} n\right) \quad\left(\bmod 2^{8}\right) .
\end{aligned}
$$

In relation (iv) of Theorem 0.2 again, we write $2^{7} n$ for $n$ and get

$$
\begin{aligned}
& 2^{7}\left[{ }_{92}^{192} p\left(2^{7} n\right)-{ }_{28}^{192} p\left(2^{7} n-12\right)\right] \\
& \quad \equiv 7 \sigma_{5}\left(2^{7} n\right)+2\left(53.2^{7} n+53\right) \sigma_{3}\left(2^{7} n\right)+\left(104.2^{14} n^{2}+28.2^{7} n+33\right) \sigma\left(2^{7} n\right) \\
& \quad \equiv 7 \sigma_{5}\left(2^{7} n\right)+106 \sigma_{3}\left(2^{7} n\right)+33 \sigma\left(2^{7} n\right) \quad\left(\bmod 2^{8}\right) .
\end{aligned}
$$

Comparing (23) and (24) we obtain

$$
{ }_{92}^{192} p\left(2^{\lambda+7} n\right)-{ }_{28}^{192} p\left(2^{\lambda+7} n-12\right) \equiv{ }_{92}^{192} p\left(2^{7} n\right)-{ }_{28}^{192} p\left(2^{7} n-12\right) \quad(\bmod 2) .
$$

And this leads in an obvious manner to the case (iv) of Corollary 2. Cases (i)-(iii) may be similarly established. In the above demonstration we have tacitly assumed $\lambda \geqq 0$, but it is easily seen that this corollary may be extended to the case (if any) where $\lambda$ is given negative values consistent with the condition that $2^{\lambda} n$ remain integral.

The above corollaries deal exclusively with the restricted partition functions. It is possible to obtain congruences involving exclusively the divisor functions $\sigma_{k}(n)$. A large number of congruences on divisor functions has been obtained by the author [6] previously.

CoRollary 3. (i) $\sigma_{3}(n) \equiv(2 n-1) \sigma(n)\left(\bmod 2^{3}\right)$;

(ii) for the sets of values $(-35,-4,-15),(5,-36,65),(45,-68,17)$ and $(53,28$, 33) of $(a, b, c)$ the following congruence is true,

$$
7 \sigma_{5}(n)+2(53 n+a) \sigma_{3}(n)+\left(104 n^{2}+b n+c\right) \sigma(n) \equiv 0 \quad\left(\bmod 2^{7}\right) .
$$

The second part of the corollary is an immediate consequence of Theorem 0.2. The first follows from a joint consideration of Theorem 0.1 and 0.2 as explained in the next section.

6. Relationship between the theorems. We shall now discuss the relationship between Theorems 0.1 and 0.2 , and also between Theorems 1 and 2. For this purpose we require the following lemma.

LEMMA 7. With respect to the modulus 2 we have

(i) ${ }_{92}^{192} p(n)-{ }_{28}^{192} p(n-12)-{ }_{68}^{192} p(n-2)+{ }_{4}^{192} p(n-22) \equiv{ }_{22}^{48} p(n)-{ }_{10}^{48} p(n-2)$,

(ii) ${ }_{76}^{192} p(n-1)-{ }_{52}^{192} p(n-5)-{ }_{44}^{192} p(n-7)+{ }_{20}^{192} p(n-15) \equiv{ }_{14}^{48} p(n-1)-{ }_{2}^{48} p(n-5)$.

These two congruences may be established by the same method, as illustrated by the following proof of the congruence (i). Obviously for $i=0,1$

$$
\sum_{v \equiv i(\bmod 4)}\left[\mp x^{v}\right]+\sum_{v \equiv i+2(\bmod 4)}\left[\mp x^{v}\right]=\sum_{v \equiv i(\bmod 2)}\left[\mp x^{v}\right] \text {. }
$$


As a consequence we have by using Lemmas 2 and 3 and confining to case $i=0$,

$$
\begin{aligned}
\sum_{-\infty}^{+\infty} x^{96 m^{2}+4 m}-\sum_{-\infty}^{+\infty} x^{96 m^{2}+28 m+2}+\sum_{-\infty}^{+\infty} x^{96 m^{2}+92 m+22}-\sum_{-\infty}^{+\infty} x^{96 m^{2}+68 m+12} \\
=\sum_{-\infty}^{+\infty} x^{24 m^{2}+2 m}-\sum_{-\infty}^{+\infty} x^{24 m^{2}+14 m+2} .
\end{aligned}
$$

Now dividing both sides by $f(x)$ and applying Lemma 4 we get

$$
\begin{aligned}
\sum_{n=0}^{\infty}{ }_{92}^{192} p(n) x^{n}-x^{2} \sum_{n=0}^{\infty}{ }_{68}^{192} p(n) x^{n} & +x^{22} \sum_{n=0}^{\infty}{ }_{492}{ }_{4} p(n) x^{n}-x^{12} \sum_{n=0}^{\infty}{ }_{28}^{192} p(n) x^{n} \\
& \equiv \sum_{n=0}^{\infty}{ }_{22}^{48} p(n) x^{n}-x^{2} \sum_{n=0}^{\infty}{ }_{10}^{48} p(n) x^{n} \quad(\bmod 2) .
\end{aligned}
$$

Relation (1) of Lemma 7 is an immediate consequence.

The two sides of the congruences in Lemma 7 may be replaced by expressions involving the divisor functions $\sigma_{k}(n)$ as given in Theorem 0.1 and 0.2 . After a little simplification we have the first part of Corollary 3 , viz.,

$$
\sigma_{3}(n) \equiv(2 n-1) \sigma(n) \quad\left(\bmod 2^{3}\right) .
$$

If the above congruence is proved independently then one can prove Theorem 0.1 from Theorem 0.2. Also Theorem 1 can be obtained from Theorem 2 with the help of Lemma 7. Either of such derivations would be very round about and complex, and we have thought fit to establish Theorems 0.1 and 1 by the simple and direct method in $\S 3$.

\section{REFERENCES}

1. L. E. Dickson, History of the theory of numbers. Vol. I, Chelsea, New York, 1952.

2. B. Gordon, A combinatorial generalization of the Rogers-Ramanujan identities, Amer. J. Math. 83 (1961), 393-399. MR 23 \#A809.

3. G. H. Hardy, Ramanujan, Cambridge Univ. Press, Cambridge and Macmillan, New York, 1940. MR 3, 71.

4. G. H. Hardy and E. M. Wright, An introduction to the theory of numbers, 4th ed., Clarendon Press, Oxford, 1960.

5. D. B. Lahiri, On a type of series involving the partition function with applications to certain congruence relations, Bull. Calcutta Math. Soc. 38 (1946), 125-132. MR 8, 445.

6. - On Ramanujan's function $\tau(n)$ and the divisor function $\sigma_{k}(n)$. I, Bull. Calcutta Math. Soc. 38 (1946), 193-206. MR 8, 567.

7. - Some restricted partition functions: Congruences modulo 7, Trans. Amer. Math. Soc. 140 (1969), 475-484.

8. S. Ramanujan, Collected papers, Cambridge Univ. Press, New York, 1927 and Chelsea, New York, 1962.

9. G. N. Watson, Über Ramanujansche Kongruenziegenschaften der Zerfälungsanzahlen, Math. Z. 39 (1935), 712-731.

\section{Indian Statistical Institute,}

CALCUTta, India 